\title{
In vitro assessment of the antifungal and paradoxical activity of different echinocandins against Candida tropicalis biofilms
}

Infections due to non-albicans Candida species are now seen with increasing frequency and in some cases their incidence exceeds that for Candida albicans (Bassetti et al., 2006). Depending on the geographical region, Candida tropicalis ranks from the second to the fourth leading cause of invasive candidiasis (Bassetti et al., 2006; Nucci et al., 2010; Pfaller et al., 2008; Tan et al., 2010). Multiple studies of echinocandins against most C. albicans biofilms have shown them to be effective at concentrations of $2 \mu \mathrm{g}$ $\mathrm{ml}^{-1}$ or less (Bachmann et al., 2002; Cocuaud et al., 2005; Ferreira et al., 2009; Jacobson et al., 2008, 2009; Kuhn et al., 2002; Miceli et al., 2009a). However, a paradoxical effect (PE) of echinocandins against $C$. albicans biofilms treated with high concentrations of caspofungin (CFG) has been described (Stevens et al., 2004). This phenomenon has subsequently been described with other echinocandins and other Candida species (Chamilos et al., 2007; Fleischhacker et al., 2008; Melo et al., 2007). This effect appears to be species-, strain- and echinocandin-specific (Chamilos et al., 2007; Ferreira et al., 2009; Melo et al., 2007; Miceli et al., 2009a, b) and associated with chitin accumulation and ultrastructural alterations (Bizerra et al., 2011); however, the clinical significance of the PE remains unclear.

There are limited comparative data available on the antifungal and paradoxical activity of anidulafungin (AFG) and micafungin (MFG) on the biofilms of non-albicans Candida species. Since C. tropicalis is becoming an emerging pathogenic Candida species, we performed a direct comparison of the efficacy and paradoxical activity of the echinocandins CFG, AFG and MFG against mature biofilms of five C. tropicalis strains.

A figure showing a comparison of the antifungal and paradoxical activity of echinocandins in Candida tropicalis biofilms is available with the online version of this paper.
One clinical (44) and four reference (ATCC 750, ATCC 44508, ATCC 44509 and ATCC 200956) strains of C. tropicalis were studied. AFG (Eraxis; Pfizer), CFG (Cancidas; Merck) and MFG (Mycamine; Astellas) were purchased from the hospital pharmacy (Raymond G. Murphy VA Medical Center). Biofilm formation and the XTT-reduction assay were performed as described by Ramage \& López-Ribot (2005). The antifungal activities of the echinocandins were expressed as a percentage relative to the metabolic activity of the untreated biofilms. Each experiment was performed independently three times, each in triplicate. The sessile (biofilm) MICs (SMICs) were determined by the concentration of each echinocandin needed to reduce the metabolic activity of the biofilms of each strain by $50 \%$ $\left(\mathrm{SMIC}_{50}\right)$ and $80 \%\left(\mathrm{SMIC}_{80}\right)$. A PE was defined as increased metabolic activity above $\mathrm{SMIC}_{50}$ despite the presence of an increasing concentration of antifungal agent.

The $\mathrm{SMIC}_{50}$ of all echinocandins for all strains tested were at concentrations of $\leqslant 1 \mu \mathrm{g} \mathrm{ml}^{-1}$ (Table 1; Supplementary Fig. $\mathrm{S} 1$ in JMM Online). The $\mathrm{SMIC}_{50}$ of CFG for all strains tested ranged from 0.25 to $1 \mu \mathrm{g} \mathrm{ml}^{-1}$. AFG and MFG had $\mathrm{SMIC}_{50}$ values of $0.25 \mu \mathrm{g} \mathrm{ml}^{-1}$ for all strains tested. The $\mathrm{SMIC}_{80}$ of CFG ranged between 1 and $2 \mu \mathrm{g} \mathrm{ml}^{-1}$. AFG had an $\mathrm{SMIC}_{80}$ of $0.25 \mu \mathrm{g}$ $\mathrm{ml}^{-1}$ for all strains except two: $1 \mu \mathrm{g} \mathrm{ml}^{-1}$ for ATCC 200956 and $128 \mu \mathrm{g} \mathrm{ml}^{-1}$ for strain 44. MFG also had an $\mathrm{SMIC}_{80}$ of $0.25 \mu \mathrm{g} \mathrm{ml}^{-1}$ for all strains except two: $0.5 \mu \mathrm{g} \mathrm{ml}^{-1}$ for ATCC 200956 and $>128 \mu \mathrm{g} \mathrm{ml}^{-1}$ for strain 44 .

A PE, i.e. increased metabolic activity at concentrations above $\mathrm{SMIC}_{50}$, was seen in three of the five strains tested. Two strains, ATCC 750 and ATCC 44508, exhibited a PE with CFG at concentrations of 32 , and 16 to $>128 \mu \mathrm{g} \mathrm{ml}^{-1}$, respectively (Table 1 ; Supplementary Fig. S1). AFG produced a PE in one strain (ATCC 200956) at concentrations from 16 to $64 \mu \mathrm{g} \mathrm{ml}^{-1}$. MFG did not exhibit a PE in any of the strains tested. No strains exhibited a PE with more than one echinocandin. While not meeting our definition of a $\mathrm{PE}$, increasing concentrations of AFG and CFG resulted in increased metabolic activity (reduced antifungal activity) above $\mathrm{SMIC}_{80}$ in all strains tested except for strain 44 for AFG and ATCC 44509 for CFG. Increasing concentrations of MFG resulted in increased metabolic activity in two strains, ATCC 44508 and ATCC 44509. Thus, despite not meeting a strict definition of PE, all strains exhibited increased metabolic activity in response to increasing concentrations of at least one echinocandin. Two strains, ATCC 750 and ATCC 220956, had increased metabolic activity with higher concentrations of all three echinocandins.

In this study, all three echinocandins were effective against C. tropicalis biofilms with the $\mathrm{SMIC}_{50}$ of all three echinocandins $\leqslant 1 \mu \mathrm{g} \mathrm{ml}^{-1}$. There was a wide range of $\mathrm{SMIC}_{50}$ values for CFG among the strains tested, compared to the $\mathrm{SMIC}_{50}$ of both AFG and MFG, which is similar to results reported by Choi et al. (2007). The $\mathrm{SMIC}_{50}$ of CFG tended to be higher than that of MFG and AFG: $1 \mu \mathrm{g} \mathrm{ml}^{-1}$ versus $0.25 \mu \mathrm{g}$ $\mathrm{ml}^{-1}$. Although our results might suggest that MFG and AFG may be more potent than CFG, it should be pointed out that the addition of human serum to AFG and MFG during in vitro susceptibility testing of Candida species sharply increased their MIC while modestly increasing the MIC of CFG (Garcia-Effron et al., 2009; Odabasi et al., 2007; Wiederhold et al., 2008). It would be of clinical interest to validate these findings within C. tropicalis biofilms.

As seen in previous studies as well as ours, CFG appears the most likely echinocandin to produce a PE whereas MFG was least likely to produce a PE in C. tropicalis biofilms. We did not see a correlation in the presence of $\mathrm{PE}$ of one echinocandin 
Table 1. $\mathrm{SMIC}_{50}, \mathrm{SMIC}_{80}$ and $\mathrm{PE}$ of the three echinocandins for all strains tested

\begin{tabular}{|c|c|c|c|c|c|c|c|c|c|c|c|c|c|c|c|}
\hline & \multicolumn{5}{|c|}{$\mathrm{SMIC}_{50}$} & \multicolumn{5}{|c|}{$\mathrm{SMIC}_{80}$} & \multicolumn{5}{|c|}{ PE (drug concentration) } \\
\hline & Strain & ATCC & ATCC & ATCC & ATCC & Strain & ATCC & ATCC & ATCC & ATCC & Strain & ATCC & ATCC & ATCC & ATCC \\
\hline & 44 & 750 & 44508 & 44509 & 200956 & 44 & 750 & 44508 & 44509 & 200956 & 44 & 750 & 44508 & 44509 & 200956 \\
\hline AFG & $<0.25$ & $<0.25$ & $<0.25$ & $<0.25$ & $<0.25$ & 0.5 & $<0.25$ & $<0.25$ & 0.25 & $<0.25$ & * & * & * & * & $\geqslant 16$ \\
\hline CFG & 1 & 0.5 & $<0.25$ & $<0.25$ & 0.5 & 2 & 1 & 1 & 1 & 1 & * & 32 & $\geqslant 16$ & - & $*$ \\
\hline MFG & $<0.25$ & $<0.25$ & $<0.25$ & $<0.25$ & $<0.25$ & 4 & 0.25 & $<0.25$ & 0.25 & $<0.25$ & * & $\star$ & - & - & * \\
\hline
\end{tabular}

*These strains did not meet our definition of a PE; however, biofilm metabolic activity increased at higher concentrations of the drug tested.

with another in any of the strains tested, or a correlation between $\mathrm{SMIC}_{50}$ and the presence of PE. However, in all the strains that exhibited a PE with one echinocandin, there was increased activity above the $\mathrm{SMIC}_{80}$ with another echinocandin.

Although the clinical significance of PE is not clear, this could theoretically have an impact on the potential use of echinocandins as a part of antimicrobial lock therapy (ALT) for the management of infected intravascular catheters. Because of the lack of adequate data, no recommendations can be made on the use of ALT in the management of catheterrelated bloodstream infections due to Candida species at this time but further studies are warranted.

\section{Acknowledgements}

This work was supported in part by grants from the Department of Veterans' Affairs (MERIT Award to S.A.L.) and the Biomedical Research Institute of New Mexico (S. A. L.).

Tsun Sheng N. Ku, ${ }^{1,2}$

Stella M. Bernardo ${ }^{1,2}$ and

Samuel A. Lee ${ }^{1,2}$

${ }^{1}$ Section of Infectious Diseases, New Mexico Veterans Healthcare System, Albuquerque, NM, USA

${ }^{2}$ Division of Infectious Diseases, University of New Mexico Health Science Center, Albuquerque, NM, USA

Correspondence: Samuel A. Lee (SamALee@salud.unm.edu)

Bachmann, S. P., VandeWalle, K., Ramage, G., Patterson, T. F., Wickes, B. L., Graybill, J. R. \& López-Ribot, J. L. (2002). In vitro activity of caspofungin against Candida albicans biofilms. Antimicrob Agents Chemother 46, 3591-3596.
Bassetti, M., Righi, E., Costa, A., Fasce, R., Molinari, M. P., Rosso, R., Pallavicini, F. B. \& Viscoli, C. (2006). Epidemiological trends in nosocomial candidemia in intensive care. $B M C$ Infect Dis 6, 21.

Bizerra, F. C., Melo, A. S., Katchburian, E., Freymüller, E., Straus, A. H., Takahashi, H. K. \& Colombo, A. L. (2011). Changes in cell wall synthesis and ultrastructure during paradoxical growth effect of caspofungin on four different Candida species. Antimicrob Agents Chemother 55, 302-310.

Chamilos, G., Lewis, R. E., Albert, N. \& Kontoyiannis, D. P. (2007). Paradoxical effect of echinocandins across Candida species in vitro: evidence for echinocandin-specific and Candida species-related differences. Antimicrob Agents Chemother 51, 2257-2259.

Choi, H. W., Shin, J. H., Jung, S. I., Park, K. H., Cho, D., Kee, S. J., Shin, M. G., Suh, S. P. \& Ryang, D. W. (2007). Species-specific differences in the susceptibilities of biofilms formed by Candida bloodstream isolates to echinocandin antifungals. Antimicrob Agents Chemother 51, 1520-1523.

Cocuaud, C., Rodier, M. H., Daniault, G. \& Imbert, C. (2005). Anti-metabolic activity of caspofungin against Candida albicans and Candida parapsilosis biofilms. J Antimicrob Chemother 56, 507-512.

Ferreira, J. A., Carr, J. H., Starling, C. E., de Resende, M. A. \& Donlan, R. M. (2009). Biofilm formation and effect of caspofungin on biofilm structure of Candida species bloodstream isolates. Antimicrob Agents Chemother 53, 4377-4384.

Fleischhacker, M., Radecke, C., Schulz, B. \& Ruhnke, M. (2008). Paradoxical growth effects of the echinocandins caspofungin and micafungin, but not of anidulafungin, on clinical isolates of Candida albicans and $C$. dubliniensis. Eur J Clin Microbiol Infect Dis 27, 127-131.

Garcia-Effron, G., Park, S. \& Perlin, D. S. (2009). Correlating echinocandin MIC and kinetic inhibition of $f k s 1$ mutant glucan synthases for Candida albicans: implications for interpretive breakpoints. Antimicrob Agents Chemother 53, 112-122.
Jacobson, M. J., Piper, K. E., Nguyen, G., Steckelberg, J. M. \& Patel, R. (2008). In vitro activity of anidulafungin against Candida albicans biofilms. Antimicrob Agents Chemother 52, 2242-2243.

Jacobson, M. J., Steckelberg, K. E., Piper, K. E., Steckelberg, J. M. \& Patel, R. (2009). In vitro activity of micafungin against planktonic and sessile Candida albicans isolates. Antimicrob Agents Chemother 53, 2638-2639.

Kuhn, D. M., George, T., Chandra, J., Mukherjee, P. K. \& Ghannoum, M. A. (2002). Antifungal susceptibility of Candida biofilms: unique efficacy of amphotericin B lipid formulations and echinocandins. Antimicrob Agents Chemother 46, 1773-1780.

Melo, A. S., Colombo, A. L. \& Arthington-Skaggs, B. A. (2007). Paradoxical growth effect of caspofungin observed on biofilms and planktonic cells of five different Candida species. Antimicrob Agents Chemother 51, 3081-3088.

Miceli, M. H., Bernardo, S. M. \& Lee, S. A. (2009a). In vitro analyses of the combination of high-dose doxycycline and antifungal agents against Candida albicans biofilms. Int $J$ Antimicrob Agents 34, 326-332.

Miceli, M. H., Bernardo, S. M. \& Lee, S. A. (2009b). In vitro analysis of the occurrence of a paradoxical effect with different echinocandins and Candida albicans biofilms. Int J Antimicrob Agents 34, 500-502.

Nucci, M., Queiroz-Telles, F., Tobón, A. M., Restrepo, A. \& Colombo, A. L. (2010). Epidemiology of opportunistic fungal infections in Latin America. Clin Infect Dis 51, 561-570.

Odabasi, Z., Paetznick, V., Rex, J. H. \& Ostrosky-Zeichner, L. (2007). Effects of serum on in vitro susceptibility testing of echinocandins. Antimicrob Agents Chemother 51, 4214-4216.

Pfaller, M. A., Boyken, L., Hollis, R. J., Kroeger, J., Messer, S. A., Tendolkar, S. \& Diekema, D. J. (2008). In vitro susceptibility of invasive isolates of Candida spp. to anidulafungin, caspofungin, and micafungin: six years of global surveillance.

J Clin Microbiol 46, 150-156. 
Ramage, G. \& López-Ribot, J. L. (2005). Techniques for antifungal susceptibility testing of Candida albicans biofilms. Methods Mol Med 118, 71-79.

Stevens, D. A., Espiritu, M. \& Parmar, R. (2004).

Paradoxical effect of caspofungin: reduced activity against Candida albicans at high drug concentrations. Antimicrob Agents Chemother 48, 3407-3411.

Tan, T. Y., Tan, A. L., Tee, N. W., Ng, L. S. \&

Chee, C. W. (2010). The increased role of nonalbicans species in candidaemia: results from a 3-year surveillance study. Mycoses 53, 515-521.
Wiederhold, N. P., Grabinski, J. L, GarciaEffron, G., Perlin, D. S. \& Lee, S. A. (2008).

Pyrosequencing to detect mutations in FKS1 that confer reduced echinocandin susceptibility in Candida albicans. Antimicrob Agents Chemother 52, 4145-4148. 